\title{
fMRI-Guided Transcranial Magnetic Stimulation Reveals That the Superior Temporal Sulcus Is a Cortical Locus of the McGurk Effect
}

\author{
Michael S. Beauchamp, Audrey R. Nath, and Siavash Pasalar \\ Department of Neurobiology and Anatomy, University of Texas Health Science Center at Houston, Houston, Texas 77030
}

\begin{abstract}
A compelling example of auditory-visual multisensory integration is the McGurk effect, in which an auditory syllable is perceived very differently depending on whether it is accompanied by a visual movie of a speaker pronouncing the same syllable or a different, incongruent syllable. Anatomical and physiological studies in human and nonhuman primates have suggested that the superior temporal sulcus (STS) is involved in auditory-visual integration for both speech and nonspeech stimuli. We hypothesized that the STS plays a critical role in the creation of the McGurk percept. Because the location of multisensory integration in the STS varies from subject to subject, the location of auditory-visual speech processing in the STS was first identified in each subject with fMRI. Then, activity in this region of the STS was disrupted with single-pulse transcranial magnetic stimulation (TMS) as subjects rated their percept of McGurk and non-McGurk stimuli. Across three experiments, TMS of the STS significantly reduced the likelihood of the McGurk percept but did not interfere with perception of non-McGurk stimuli. TMS of the STS was effective at disrupting the McGurk effect only in a narrow temporal window from $100 \mathrm{~ms}$ before auditory syllable onset to $100 \mathrm{~ms}$ after onset, and TMS of a control location did not influence perception of McGurk or control stimuli. These results demonstrate that the STS plays a critical role in the McGurk effect and auditory-visual integration of speech.
\end{abstract}

\section{Introduction}

A textbook example of how both the auditory and visual modalities are important for speech perception is the McGurk effect, in which an auditory syllable (phoneme) is perceived very differently depending on whether it is accompanied by a visual movie of a speaker pronouncing the same syllable or a different, incongruent syllable (McGurk and MacDonald, 1976). The superior temporal sulcus (STS) has been implicated in auditory-visual multisensory integration for both speech and nonspeech stimuli (Calvert et al., 2000; Sekiyama et al., 2003; Beauchamp, 2005a; Miller and D'Esposito, 2005). However, neuroimaging studies that have compared incongruent auditory-visual speech, including McGurk syllables, to congruent speech or other baselines have reported differences in a broad network of brain regions in addition to the STS, including the supramarginal gyrus (Jones and Callan, 2003; Bernstein et al., 2008), the inferior parietal lobule (Jones and Callan, 2003), the precentral gyrus (Jones and Callan, 2003), the superior frontal gyrus (Miller and D'Esposito, 2005), Heschl's gyrus (Miller and D'Esposito, 2005), and the

Received Sept. 30, 2009; revised Dec. 17, 2009; accepted Dec. 23, 2009.

This research was supported by National Science Foundation Cognitive Neuroscience Initiative Research Grant 0642532 to M.S.B. S.P. was supported by National Institutes of Health (NIH) Grant T32HD049350 (Harvey S. Levin, Principal Investigator). A.N. was supported by NIH Grant TL1RR024147. Partial funding for purchase of the MR scanner was provided by NIH Grant S10 RR19186. We thank Tony Ro and Nafi Yasar for assistance with TMS and Vips Patel for assistance with MR data collection.

Correspondence should be addressed to Michael S. Beauchamp, Department of Neurobiology and Anatomy, University of Texas Health Science Center at Houston, 6431 Fannin Street, Suite G.550, Houston, TX 77030. E-mail: Michael.S.Beauchamp@uth.tmc.edu.

DOI:10.1523/JNEUROSCI.4865-09.2010

Copyright $\odot 2010$ the authors $\quad 0270-6474 / 10 / 302414-04 \$ 15.00 / 0$ middle temporal gyrus (Callan et al., 2004). A recent fMRI study even claims that the STS is not especially important for auditoryvisual integration (Hocking and Price, 2008). Interpreting these conflicting results is problematic because the blood oxygenation level-dependent (BOLD) signal is an indirect measure of neural activity with poor temporal resolution, limiting inferences about whether activity in an area is causally related to (or merely correlated with) a particular cognitive process. For instance, frontal areas could respond to incongruity in the stimulus but not be responsible for the dramatically altered percept that defines the McGurk effect.

Transcranial magnetic stimulation (TMS) can be used to create a temporary virtual lesion and assess the necessity of a brain area for perception; delivering single-pulse TMS at various latencies allows the time window in which the area contributes to perception to be determined (Pascual-Leone et al., 2000; Walsh and Cowey, 2000). However, TMS stimulation of cortical association areas such as the STS does not produce phosphenes (unlike V1) or motor twitches (unlike M1), making localization by TMS ineffective. Because there is substantial intersubject variability in the anatomical location of the STS multisensory area (Beauchamp et al., 2004a), it is difficult to target the STS and other association areas with skull-based landmarks (Sack et al., 2009). This has spurred the development of methods that combine fMRI and TMS in individual subjects (Andoh et al., 2006; Sack et al., 2006; Sparing et al., 2008). Using these methods, we created temporary lesions in the STS and found a dramatic reduction in the likelihood of the McGurk effect, demonstrating that the STS is a cortical locus for the McGurk effect and auditory-visual integration in speech. 


\section{Materials and Methods}

Experiments were conducted in accordance with the Committee for the Protection of Human Subjects of the University of Texas Health Science Center at Houston. Twelve healthy volunteers (four females, two lefthanded, mean age 25 years) with no history of neurological or sensory disorders participated in the study (nine in experiment 1 , nine in experiment 2, six in experiment 3). Subjects were screened for the exclusion criteria for MRI and TMS and written informed consent was obtained from each subject before experimentation.

Stimuli. McGurk and non-McGurk stimuli were digitally recorded and edited (see supplemental materials, available at www.jneurosci.org, for a sample stimulus). In experiments 1 and 3 , a male speaker was used. The McGurk stimulus consisted of an auditory recording of "ba" and a digital video of the speaker enunciating "ga." This produced the McGurk effect percept of "da." The control stimulus consisted of an auditory recording of "ba" and a gray screen with white fixation crosshairs (but no digital video) producing the percept "ba." To demonstrate that the disruption of the McGurk effect was robust to stimulus changes, in experiment 2 a different stimulus set was used with a female speaker. The McGurk stimulus consisted of an auditory recording of "pa" and a digital video of the speaker enunciating "ka" or "na," producing the McGurk effect percept of "ta" (Sekiyama, 1994). The control stimulus consisted of an auditory recording of "pa" and a digital video of the speaker enunciating "pa," producing the percept "pa." Some subjects do not exhibit the McGurk effect (MacDonald et al., 2000). Therefore, an informal screening was used at the first stage of subject recruitment. Four subjects who did not report the McGurk percept were excluded; the remaining 12 subjects participated in the experiments.

TMS. During the TMS experiments, seated subjects viewed visual stimuli on an LCD screen placed at eye level $65 \mathrm{~cm}$ from the subject. In-ear headphones were used to deliver auditory speech stimuli and subjects made button-press responses with their left hand. A biphasic TMS unit (Magstim Rapid; Magstim) with a $70 \mathrm{~mm}$ figure-of-eight coil was used to deliver TMS. The coil was positioned using an image-guided neuro-navigation system for frameless stereotaxy (Brainsight; Rogue Research). The stimulation site was plotted as the coordinate on the surface of the brain closest to the TMS coil, calculated as the position where a line normal to the surface of the scalp first intersects the brain surface. The motor threshold intensity was determined for each subject (on average $68 \pm 2 \%$ of machine output) and used throughout the session (Ro et al., 2004; Stokes et al., 2005; Balslev et al., 2007).

In experiments 1 and 2, each run consisted of 10 trials of randomly intermixed McGurk and control stimuli with and without TMS for a total of 40 trials. Each subject completed two runs, one with the TMS coil targeting the left STS and one with the TMS coil targeting a control site. Single-pulse TMS was delivered at the onset of the frame of the video nearest to the onset of the auditory syllable (experiment 1: $1155 \mathrm{~ms}$ after visual stimulus onset, $32 \mathrm{~ms}$ after auditory onset; experiment 2: $528 \mathrm{~ms}$ after visual onset, $19 \mathrm{~ms}$ after auditory onset).

In experiment 3 , single-pulse TMS was delivered to the STS in every trial at one of 11 times from $298 \mathrm{~ms}$ before to $362 \mathrm{~ms}$ after auditory onset, with step size $66 \mathrm{~ms}$ (corresponding to two video frames). As in experiments 1 and 2, there were 10 trials per condition for a total of 110 trials.

MRI. Anatomical MRI scans were obtained from each subject using a 3 tesla whole-body MR scanner (Philips Medical Systems) using a 16channel head gradient coil. Images were collected using a magnetizationprepared, 180 degree radio frequency pulses and rapid-gradient echo sequence optimized for gray-white matter contrast with 1-mm-thick sagittal slices and an in-plane resolution of $0.938 \times 0.938 \mathrm{~mm}$. AFNI software (Cox, 1996) was used to analyze MRI data. Three-dimensional cortical surface models were created with FreeSurfer (Fischl et al., 1999) and visualized in SUMA (Argall et al., 2006). Cortical surfaces were partially inflated using 500 iterations of a smoothing algorithm to better visualize the deeper sulcal areas (Van Essen, 2004). To allow reporting of the stimulation sites in standard coordinates, each individual subject brain was normalized to the N27 atlas brain (Mazziotta et al., 2001). For five subjects, the STS multisensory area was localized using an anatomical landmark: the inflection point in the posterior STS where it angles up- ward toward the parietal lobe (Beauchamp et al., 2008). For the remaining seven subjects, the STS multisensory area was localized using BOLD fMRI. Functional images were collected using gradient echo, echo planar imaging $\left(\mathrm{TR}=2015 \mathrm{~ms}, \mathrm{TE}=30 \mathrm{~ms}\right.$, flip angle $\left.=90^{\circ}\right)$ with a voxel size of $3 \times 2.75 \times 2.75 \mathrm{~mm}$. The localizer consisted of one $150 \mathrm{TR}$ run with audio and video stimulus blocks. One hundred single-syllable words from the MRC Psycholinguistic Database (Wilson, 1988) were spoken by a female speaker. Auditory stimuli consisted of auditory words presented during visual presentation of crosshairs. Visual stimuli consisted of word videos with no accompanying sound. Five blocks of auditory words and five blocks of visual words were presented in random order. Three subjects were also presented with blocks of simultaneous auditory (A) and visual $(\mathrm{V})$ words. Each block consisted of 10 consecutive stimuli, one per TR, followed by five TRs of fixation with crosshairs at the approximate location of the mouth. To reduce any visual afterimage, a static image of a scrambled face was presented for $50 \mathrm{~ms}$ after each video. Visual stimuli were projected onto a screen and viewed by the subject using a mirror. MRI-compatible pneumatic headphones were used to present auditory stimuli inside the scanner. During the course of scanning, MRcompatible eye tracking (Applied Science Laboratories) was used to ensure arousal and attention. Stimuli were presented using Presentation software (Neurobehavioral Systems). The general linear model was used to detect voxels showing a significant response to auditory or visual speech, followed by a conjunction analysis of auditory $\cap$ visual activation. An automated parcellation routine determined all $\mathrm{A} \cap \mathrm{V}$ voxels in the STS (Fischl et al., 2004; Beauchamp et al., 2008). The center of mass of the resulting cluster of active voxels was used as the target site for STS TMS. Different statistical criteria for classifying multisensory voxels in the STS, such as the mean response to audiovisual (AV) words or the contrast of AV versus mean $(A, V)$, changed the extent but had little impact on the center of mass of the STS multisensory area (Beauchamp, 2005b).

\section{Results}

Consistent with previous studies, the fMRI localizers revealed a focus of activity in the posterior STS that responded to both auditory and visual speech (Fig. $1 A$ ). There was a high degree of intersubject variability in the standard coordinates of the STS multisensory area, especially in the anterior-to-posterior direction $[x=-56 \pm 4 \mathrm{~mm} ; y=-27 \pm 12 \mathrm{~mm} z=8 \pm 9 \mathrm{~mm}$ $($ mean $\pm \mathrm{SD})]$.

To verify the effectiveness of our stimuli, we examined subjects' percepts without TMS (Fig. $1 B, C$ ). The non-McGurk control stimuli consisted of an auditory syllable (experiment 1 ) or a congruent auditory-visual syllable (experiment 2 ). When presented with these stimuli, subjects almost always reported a percept that matched the auditory stimulus (mean likelihoods and reaction times in Table 1). The McGurk stimuli consisted of an incongruent auditory-visual syllable. For these stimuli, subjects rarely reported a percept that matched the auditory stimulus, instead reporting the McGurk percept of a fused syllable different from both the auditory and visual syllables.

When TMS was delivered to the STS, subjects were significantly less likely to report the McGurk effect (experiment 1, $p=0.00005$; experiment $2, p=0.004$ ). A concern was that nonspecific effects of TMS could introduce a possible confound. For instance, the brief click of the TMS pulse could somehow interfere with auditory perception. To address this concern, we stimulated a control TMS site dorsal and posterior to the STS, producing a similar behavioral experience for the subject. The mean coordinates of the control site in standard space were $(x, y, z)=(-42,-19,46)$ a distance of $39 \pm 12 \mathrm{~mm}(\mathrm{SD})$ from the STS site $(-60,-35,16)$. TMS of the control site did not reduce the likelihood of perceiving the McGurk effect (experiment $1, p=0.2$; experiment $2, p=0.5$ ). A second concern was that TMS of the STS might interfere with speech perception in general. However, TMS of the STS did not affect discrimination 

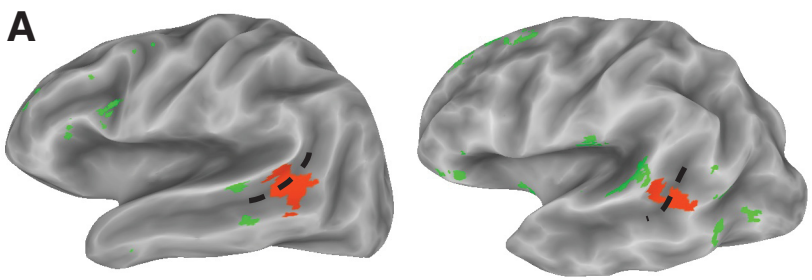

B

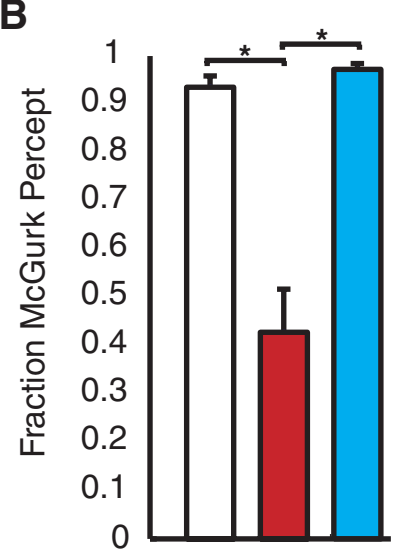

C
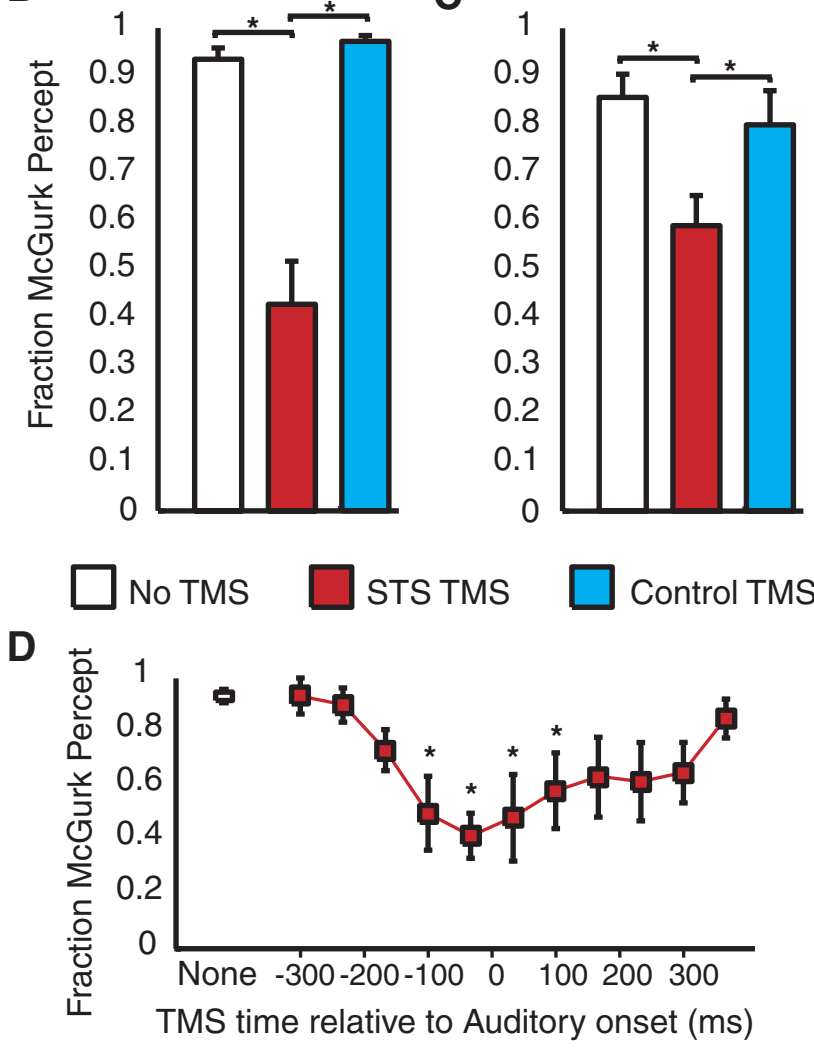

Figure 1. $\mathrm{fMRI}$-guided TMS of congruent and incongruent auditory-visual speech. $\boldsymbol{A}$, Presentation of auditory-visual speech produced strong activation in the left superior temporal sulcus, shown in the partially inflated left hemisphere of two individual subjects. Colored regions show cortex active during both auditory and visual speech perception. Orange color highlights active cortex in the STS; green shows active cortex outside the STS. The dashed black line shows the fundus of the posterior STS. $\boldsymbol{B}$, During presentation of the experiment 1 McGurk stimulus, subjects were likely to report the McGurk percept (white bar); TMS of the STS (red bar) but not control TMS (blue bar) significantly reduced the likelihood $\left({ }^{*} p<0.05\right)$. Error bars show SEM across subjects. C, During presentation of the experiment 2 McGurk stimulus, subjects were likely to report the McGurk percept; TMS of the STS but not control TMS reduced the likelihood. D, The effectiveness of single-pulse TMS of the STS depended on the time at which it was delivered. TMS delivered near the onset of the auditory stimulus reduced the likelihood of the McGurk percept but TMS at other times did not.

of the control stimuli (experiment $1, p=0.5$; experiment $2, p=$ 0.3 ). If multisensory integration in the STS is the basis of the McGurk effect, the relevant neural computation must occur in a relatively narrow time window after the auditory and visual stimuli are delivered but before perception occurs. To test this idea, in the third experiment the likelihood of the McGurk effect was measured while single-pulse TMS was delivered to the STS at a range of times. There was a significant effect of stimulation time on the McGurk effect $\left[F_{(10,50)}=4.66, p=0.0001\right]$. This was driven by a reduction in the McGurk effect at four time points, spanning $100 \mathrm{~ms}$ before onset of the auditory stimuli to $100 \mathrm{~ms}$ after onset of the auditory stimulus $(p<0.05$ by Mann-Whitney
Table 1. Likelihood of the McGurk percept (\%) and the reaction time (ms) ( \pm SEM) for each condition of experiments 1 and 2

\begin{tabular}{cccc}
\hline & No TMS & STS TMS & Control TMS \\
\hline Experiment 1 & & & \\
Audio-only & $1 \pm 1 \%$ & $3 \pm 3 \%$ & $6 \pm 3 \%$ \\
& $1089 \pm 206 \mathrm{~ms}$ & $1221 \pm 230 \mathrm{~ms}$ & $1166 \pm 222 \mathrm{~ms}$ \\
McG AV & $94 \pm 2 \%$ & $43 \pm 9 \%$ & $97 \pm 1 \%$ \\
& $1048 \pm 177 \mathrm{~ms}$ & $1386 \pm 255 \mathrm{~ms}$ & $1073 \pm 189 \mathrm{~ms}$ \\
Experiment 2 & & & \\
Cong AV & $1 \pm 1 \%$ & $3 \pm 2 \%$ & $2 \pm 2 \%$ \\
& $907 \pm 105 \mathrm{~ms}$ & $1021 \pm 179 \mathrm{~ms}$ & $743 \pm 66 \mathrm{~ms}$ \\
McG AV & $86 \pm 5 \%$ & $59 \pm 6 \%$ & $80 \pm 7 \%$ \\
& $1210 \pm 166 \mathrm{~ms}$ & $1496 \pm 265 \mathrm{~ms}$ & $1037 \pm 157 \mathrm{~ms}$ \\
\hline
\end{tabular}

Audio-only, auditory-only control stimulus; McG AV, McGurk auditory-visual stimulus; Cong AV, congruent auditoryvisual control stimulus.

$U$ test). At other times, STS TMS did not significantly change the McGurk percept (Fig. 1D).

Subjects reported a variety of percepts during auditory-visual trials in which STS TMS disrupted the McGurk effect. The most common experience, reported $66 \%$ of the time, was a percept similar to auditory-only trials (e.g., TMS delivered with auditory "ba" plus visual "ga" resulted in the percept "ba" instead of the McGurk percept "da"). The second most common experience was a percept between the auditory and McGurk percepts (e.g., between "ba" and "da"). Other reports were of a hybrid percept (e.g., "b-da") or a completely different syllable (e.g., "ha").

\section{Discussion}

These experiments demonstrate that temporary disruption of the STS with TMS causes a dramatic reduction in the perception of the McGurk effect. The role of the STS in auditory-visual integration has been called into question because the STS also shows fMRI responses during other tasks, such as visual-visual associations (Hocking and Price, 2008). However, BOLD fMRI is an indirect method of measuring neural activity with limited temporal precision. TMS allows for the perturbation of brain areas to demonstrate a causal link between brain and behavior (PascualLeone et al., 2000; Walsh and Cowey, 2000). Combining TMS with anatomical and functional MRI allows the same functional brain region to be targeted in each subject, greatly increasing the statistical power of TMS studies of association areas, like the STS multisensory area, that are difficult to accurately localize with other methods (Sparing et al., 2008; Sack et al., 2009). A privileged role for the STS in auditory-visual integration is demonstrated by the finding that temporary disruption of the STS interferes with the McGurk effect, which depends on the interaction between the auditory and visual modalities.

TMS of the STS disrupted the McGurk effect only when single-pulse TMS was delivered in a $200 \mathrm{~ms}$ window spanning 100 $\mathrm{ms}$ before to $100 \mathrm{~ms}$ after auditory stimulus presentation, supporting the notion that TMS disrupts a specific neural computation in the STS-auditory-visual integration-that is time-locked to stimulus presentation. This finding is consistent with behavioral results showing that the McGurk effect is robust to auditoryvisual asynchronies within a $200 \mathrm{~ms}$ integration window (van Wassenhove et al., 2007) and results from electrophysiological recording demonstrating strong responses in STS beginning $\sim 100 \mathrm{~ms}$ after stimulus presentation in monkeys (Schroeder and Foxe, 2002; Barraclough et al., 2005) and humans (Canolty et al., 2007; Puce et al., 2007). TMS disruption of the STS after the auditory and visual syllables have been integrated should not, and did not, affect perception. 
What is the neuronal architecture of the STS that produces the McGurk effect? High-resolution fMRI and single-unit recording studies have shown that cortex in the STS contains a patchy distribution of neurons that respond to auditory, visual, or auditoryvisual stimuli (Beauchamp et al., 2004b; Dahl et al., 2009). Multivoxel pattern analysis of BOLD fMRI data has demonstrated that activity in a region that includes the STS can discriminate between individual auditory syllables (e.g., "ba" vs "da") (Formisano et al., 2008; Raizada et al., 2009). These results suggest an architecture in which the STS contains small patches of neurons that respond to specific syllables. Activity across multiple syllable patches would be compared using a winner-take-all algorithm, with the most active patch determining perception. Each patch might receive input from neurons in visual and auditory association areas coding for specific visemes and phonemes. During presentation of congruent auditory-visual speech, input from auditory and visual neurons would be integrated, improving sensitivity. During presentation of incongruent McGurk stimuli, this process could result in unexpected percepts. For instance, if an STS patch representing "da" received input from both auditory "ba" and visual "ga" neurons, the patch would have a large response during presentation of the "ba" plus "ga" McGurk stimulus, producing a "da" percept. Disrupting activity in the STS would eliminate this multisensory integration. In future studies, it will be important to test this model and refine our understanding of the temporal and spatial organization of multisensory responses in the STS.

\section{References}

Andoh J, Artiges E, Pallier C, Rivière D, Mangin JF, Cachia A, Plaze M, Paillere-Martinot ML, Martinot JL (2006) Modulation of language areas with functional MR image-guided magnetic stimulation. Neuroimage 29:619-627.

Argall BD, Saad ZS, Beauchamp MS (2006) Simplified intersubject averaging on the cortical surface using SUMA. Hum Brain Mapp 27:14-27.

Balslev D, Braet W, McAllister C, Miall RC (2007) Inter-individual variability in optimal current direction for transcranial magnetic stimulation of the motor cortex. J Neurosci Methods 162:309-313.

Barraclough NE, Xiao D, Baker CI, Oram MW, Perrett DI (2005) Integration of visual and auditory information by superior temporal sulcus neurons responsive to the sight of actions. J Cogn Neurosci 17:377-391.

Beauchamp MS (2005a) See me, hear me, touch me: multisensory integration in lateral occipital-temporal cortex. Curr Opin Neurobiol 15:145-153.

Beauchamp MS (2005b) Statistical criteria in FMRI studies of multisensory integration. Neuroinformatics 3:93-113.

Beauchamp MS, Lee KE, Argall BD, Martin A (2004a) Integration of auditory and visual information about objects in superior temporal sulcus. Neuron 41:809-823.

Beauchamp MS, Argall BD, Bodurka J, Duyn JH, Martin A (2004b) Unraveling multisensory integration: patchy organization within human STS multisensory cortex. Nat Neurosci 7:1190-1192.

Beauchamp MS, Yasar NE, Frye RE, Ro T (2008) Touch, sound and vision in human superior temporal sulcus. Neuroimage 41:1011-1020.

Bernstein LE, Lu ZL, Jiang J (2008) Quantified acoustic-optical speech signal incongruity identifies cortical sites of audiovisual speech processing. Brain Res 1242:172-184.

Callan DE, Jones JA, Munhall K, Kroos C, Callan AM, Vatikiotis-Bateson E (2004) Multisensory integration sites identified by perception of spatial wavelet filtered visual speech gesture information. J Cogn Neurosci 16:805-816.

Calvert GA, Campbell R, Brammer MJ (2000) Evidence from functional magnetic resonance imaging of crossmodal binding in the human heteromodal cortex. Curr Biol 10:649-657.

Canolty RT, Soltani M, Dalal SS, Edwards E, Dronkers NF, Nagarajan SS, Kirsch HE, Barbaro NM, Knight RT (2007) Spatiotemporal dynamics of word processing in the human brain. Front Neurosci 1:185-196.
Cox RW (1996) AFNI: software for analysis and visualization of functional magnetic resonance neuroimages. Comput Biomed Res 29:162-173.

Dahl CD, Logothetis NK, Kayser C (2009) Spatial organization of multisensory responses in temporal association cortex. J Neurosci 29:11924-11932.

Fischl B, Sereno MI, Dale AM (1999) Cortical surface-based analysis. II. Inflation, flattening, and a surface-based coordinate system. Neuroimage 9:195-207.

Fischl B, van der Kouwe A, Destrieux C, Halgren E, Ségonne F, Salat DH, Busa E, Seidman LJ, Goldstein J, Kennedy D, Caviness V, Makris N, Rosen B, Dale AM (2004) Automatically parcellating the human cerebral cortex. Cereb Cortex 14:11-22.

Formisano E, De Martino F, Bonte M, Goebel R (2008) "Who" is saying "what"? Brain-based decoding of human voice and speech. Science 322:970-973.

Hocking J, Price CJ (2008) The role of the posterior superior temporal sulcus in audiovisual processing. Cereb Cortex 18:2439-2449.

Jones JA, Callan DE (2003) Brain activity during audiovisual speech perception: an fMRI study of the McGurk effect. Neuroreport 14:1129-1133.

MacDonald J, Andersen S, Bachmann T (2000) Hearing by eye: how much spatial degradation can be tolerated? Perception 29:1155-1168.

Mazziotta J, Toga A, Evans A, Fox P, Lancaster J, Zilles K, Woods R, Paus T, Simpson G, Pike B, Holmes C, Collins L, Thompson P, MacDonald D, Iacoboni M, Schormann T, Amunts K, Palomero-Gallagher N, Geyer S, Parsons L, et al (2001) A probabilistic atlas and reference system for the human brain: International Consortium for Brain Mapping (ICBM). Philos Trans R Soc Lond B Biol Sci 356:1293-1322.

McGurk H, MacDonald J (1976) Hearing lips and seeing voices. Nature 264:746-748

Miller LM, D'Esposito M (2005) Perceptual fusion and stimulus coincidence in the cross-modal integration of speech. J Neurosci 25:5884-5893.

Pascual-Leone A, Walsh V, Rothwell J (2000) Transcranial magnetic stimulation in cognitive neuroscience-virtual lesion, chronometry, and functional connectivity. Curr Opin Neurobiol 10:232-237.

Puce A, Epling JA, Thompson JC, Carrick OK (2007) Neural responses elicited to face motion and vocalization pairings. Neuropsychologia 45:93-106.

Raizada RD, Tsao FM, Liu HM, Kuhl PK (2010) Quantifying the adequacy of neural representations for a cross-language phonetic discrimination task: prediction of individual differences. Cereb Cortex 20:1-12.

Ro T, Wallace R, Hagedorn J, Farnè A, Pienkos E (2004) Visual enhancing of tactile perception in the posterior parietal cortex. J Cogn Neurosci 16:24-30.

Sack AT, Kohler A, Linden DE, Goebel R, Muckli L (2006) The temporal characteristics of motion processing in hMT/V5+: combining fMRI and neuronavigated TMS. Neuroimage 29:1326-1335.

Sack AT, Cohen Kadosh R, Schuhmann T, Moerel M, Walsh V, Goebel R (2009) Optimizing functional accuracy of TMS in cognitive studies: a comparison of methods. J Cogn Neurosci 21:207-221.

Schroeder CE, Foxe JJ (2002) The timing and laminar profile of converging inputs to multisensory areas of the macaque neocortex. Brain Res Cogn Brain Res 14:187-198.

Sekiyama K (1994) McGurk effect and incompatibility: a cross-language study on auditory-visual speech perception: studies and essays. Behav Sci Philos 14:29-62.

Sekiyama K, Kanno I, Miura S, Sugita Y (2003) Auditory-visual speech perception examined by fMRI and PET. Neurosci Res 47:277-287.

Sparing R, Buelte D, Meister IG, Paus T, Fink GR (2008) Transcranial magnetic stimulation and the challenge of coil placement: a comparison of conventional and stereotaxic neuronavigational strategies. Hum Brain Mapp 29:82-96.

Stokes MG, Chambers CD, Gould IC, Henderson TR, Janko NE, Allen NB, Mattingley JB (2005) Simple metric for scaling motor threshold based on scalp-cortex distance: application to studies using transcranial magnetic stimulation. J Neurophysiol 94:4520-4527.

Van Essen DC (2004) Surface-based approaches to spatial localization and registration in primate cerebral cortex. Neuroimage 23[Suppl 1]:S97-S107.

van Wassenhove V, Grant KW, Poeppel D (2007) Temporal window of integration in auditory-visual speech perception. Neuropsychologia 45:598-607.

Walsh V, Cowey A (2000) Transcranial magnetic stimulation and cognitive neuroscience. Nat Rev Neurosci 1:73-79.

Wilson M (1988) The MRC psycholinguistic database: machine readable dictionary, Version 2. Behav Res Methods Instrum Comput 20:6-11. 